\title{
Perturbation of BRMS1 interactome reveals pathways that impact cell migration
}

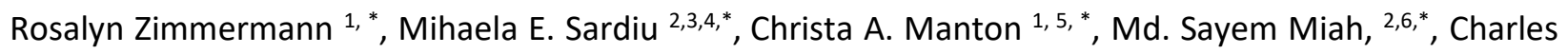
A.S. Banks ${ }^{2}$, Mark K. Adams ${ }^{2}$, Devin C. Koestler ${ }^{3,4}$, Michael P. Washburn 1,2,4, Danny R. Welch ${ }^{1,4}$

${ }^{1}$ Department of Cancer Biology, The Kansas University Medical Center, 3901 Rainbow Blvd., Kansas City, KS, 66160, USA

${ }^{2}$ Stowers Institute for Medical Research, Kansas City, Missouri 64110, USA.

${ }^{3}$ Department of Biostatistics and Data Science, The Kansas University Medical Center, 3901 Rainbow Blvd., Kansas City, KS, 66160, USA

${ }^{4}$ The University of Kansas Cancer Center, 3901 Rainbow Blvd., Kansas City, KS 66160, USA

Address correspondence to:

Danny R. Welch, PhD, Department of Cancer Biology, The Kansas University Medical Center, 3901 Rainbow Blvd., Kansas City, KS, 66160 Email: dwelch@kumc.edu

Current address:

${ }^{5}$ Department of Biology, Baker University, 618 8th St, Baldwin City, KS 66006

${ }^{6}$ Department of Biochemistry and Molecular Biology, University of Arkansas for Health Sciences, Little Rock, AR 72205

${ }^{*}$ Contributed equally to this work 


\begin{abstract}
Breast Cancer Metastasis Suppressor 1 (BRMS1) expression has been associated with longer patient survival in multiple cancer types. Understanding BRMS1 at the protein level will provide insights into both mechanism of action and enhance potential therapeutic development. We previously mapped the Cterminus of BRMS1 as critical for metastasis suppression and hypothesized that critical protein interactions in this region will explain function. These studies indicate that phosphorylation status at S237 regulates BRMS1 interactions related to a variety of biological processes, phenotypes [cell cycle (e.g., CDKN2A), DNA repair (e.g., BRCA1)], and metastasis [(e.g., TCF2 and POLE2)]. Presence of the C-terminal site appears to be critical for BRMS1 directed metastasis suppression, as demonstrated by in vitro migration assays. These assays demonstrated that presence of S237 directly decreased MDA-MB-231 migration. This study furthers our understanding of BRMS1's molecular role, as it demonstrates that BRMS1 C-terminus is involved in direct protein-protein interactions. Several of the interacting proteins are associated with cancer and metastasis, which may result in metastasis suppression as suggested by in vitro findings.
\end{abstract}

Keywords: BRMS1/metastasis/cancer/SIN3 HDAC complex

\title{
Introduction
}

Metastasis is a multi-step process that occurs when cells disseminate from the primary neoplasm and eventually colonize distant organs. Successful completion of this complex cascade is associated with 90 percent of cancer-related morbidities and mortalities. Despite its causative role in cancer-specific mortality and morbidity, a complete understanding of the process and its mechanisms remain elusive. Metastasis is regulated by three types of genes: metastasis-promoting, -suppressing, and -efficiency modifying [1]. The protein of interest in this study, Breast Cancer Metastasis Suppressor 1 (BRMS1), is characterized as a metastasis suppressor, which are defined by their ability to suppress metastasis without blocking primary tumor growth [2]. They are of distinct interest because, by preventing the successful completion of the metastatic cascade, the devastating sequalae or deaths associated with metastasis are inhibited $[3,4]$.

BRMS1 was discovered in 2000 and its re-expression in multiple cell lines significantly decreases lung metastases in mice [5-8]. In addition, higher expression of BRMS1 in breast, lung, and melanoma cancers 
is associated with improved patient survival [8-10]. Despite promising functional evidence and clinical correlations, the mechanism by which BRMS1 blocks metastasis is yet to be understood.

BRMS1 is a known member of $\operatorname{Sin} 3$ histone deacetylase (HDAC) transcriptional regulatory complexes in multiple eukaryotic cell types [11-13]. It is an established binding partner of SUDS3 and ARID4A and is also associated with several other members of the complex such as SIN3A, SIN3B, HDAC1, and HDAC2 [11]. Relatedly, BRMS1 also exhibits E3 ubiquitin ligase function on the histone acetyl transferase p300, which could also regulate its metastatic function [14]. Cumulatively, it appears that BRMS1 regulation of transcription via epigenetic pathways is involved in metastasis suppression; however, further studies must be completed to ascribe a definitive mechanism of action.

In our quest to translate BRMS1 into clinical practice, additional structural data are necessary. This study leverages previously described features of BRMS1 to clarify its molecular functions within a cell. Previous attempts to define BRMS1 structure-function have been met with uneven success. To date no one has successfully crystallized and obtained structure for full-length, wild-type BRMS1. But computer algorithms and NMR studies have identified structural information for BRMS1 domains [15]. BRMS1 has predicted protein domains that consists of glutamate-rich regions (aa 1-50), two coiled-coil domains (aa 51-81, aa 147-180), and a nuclear localization sequence (aa 198-205) [5]. The $\mathrm{N}$-terminus has been characterized and its 3D structure crystalized $[16,17]$, but outside of amino acids 51-98 there is no precise protein structure for the entirety of the 246 amino acid BRMS1 protein. The C-terminus is of particular interest as it has been shown to play a critical role in metastatic suppression, due to in vivo studies demonstrating that alteration of the domain impacts overall metastatic burden [18]. Of note, the serine immediately upstream of the critical metastasis suppressor domain was found to be phosphorylated by Cyclin Dependent Kinase 2 (CDK2) [19]. Due to the juxtaposition of S237 to the C-terminal metastasis suppression region, this study focused on defining the C-terminus structure as well as identifying proteins with which BRMS1 interacts in that region, the results of which can be used to better understand how BRMS1 may function to suppress metastasis. 


\section{Results and Discussion}

\section{BRMS1 C-terminal protein structure is important for protein-protein interactions, including Sin3/HDAC} complex interaction. Given the lack of crystal structure for BRMS1 (and specifically the C-terminus), we utilized a protein structure prediction approach within I-TASSER (Figure 1A and 1B). The algorithmpredicted structure demonstrates that the C-terminal region consists of helical, coiled, and strand regions, and the phosphorylation site of interest lies within a coiled domain (Figure 1A). The predicted coiled domain, coupled with a previous classification of this region as intrinsically disordered - both of which are associated with protein-protein interactions - supported our strategy to hypothesize that the region may play a role in protein binding as a mechanism by which it functions to suppress metastasis [15].

Utilizing recently published crosslinking data of Sin3/HDAC complex members [12], BRMS1 was confirmed to interact with its known interaction partner SUDS3 (at BRMS1 aa 142), while also binding to the Sin3/HDAC member HDAC1 at BRMS1 aa 184 (Figure 1C). BRMS1 also cross-links with SIN3A but not with the related SIN3B (Figure 1C). Importantly, the location of SIN3A binding is primarily at BRMS1's Cterminus (at BRMS1 aa 201, 240, and 242) (Figure 1C). Together, these data suggest that BRMS1 may regulate the transcriptional profile of SIN3A, but not SIN3B. The implications of these findings are twofold. The location of the binding within the C-terminus supports the hypothesis that the C-terminus is involved in protein binding and may regulate BRMS1's binding partners. Furthermore, the proximity of the binding sites (aa 240, 242) near S237 further supports the hypothesis that S237 phosphorylation plays a role in regulating BRMS1 binding partners. Additionally, this data further defines BRMS1's role within the $\operatorname{Sin} 3 /$ HDAC complexes.

Combining the previously published functional studies, interaction studies and these new structural predictions, we generated a panel of BRMS1 mutants: BRMS1 ${ }^{\text {S237D }}$ (phosphorylation-mimic, hereafter S237D); BRMS1 ${ }^{\text {S237A }}$ (unable to be phosphorylated, hereafter S237A); BRMS1 ${ }^{1-229}$ (lacks critical C-terminal domain); and BRMS1 ${ }^{230-246}$ (C-terminal domain with S237 in the center) (Figure 2A). Regardless of whether S237 was mutated to aspartic acid or alanine, BRMS1 protein stability was predicted to be compromised using multiple in silico prediction methods (Table 1). These data suggest that S237 site modifications dramatically destabilize BRMS1 and may be important for overall protein function.

\section{BRMS1 binds Sin3/HDAC complex members.}

Based upon the combination of findings within I-TASSER and in silico prediction methods suggesting BRMS1 C-terminus functions in protein binding, we posited that the phosphorylation site could 
regulate binding. To test this hypothesis, mass spectrometry was completed (Supplementary Table 1) in 293T cells transduced with BRMS1 wild-type $\left(B R M S 1^{\text {WT }}\right.$ ), BRMS1 ${ }^{1-229}$, S237A, S237D, or BRMS1 $230-246$ (Figure 2A). Constructs were selected because they allowed focus on both the phosphorylation as well as putative protein interaction location(s). This point is key as BRMS1 contains two coiled-coiled domains which are within $\mathrm{BRMS}^{1-229}$ but which are lacking in $\mathrm{BRMS} 1^{230-246}$, allowing us to identify proteins binding specific to each region.

Mass spectrometry identified 1175 proteins whose binding was significantly (QSPEC Z-Score $\leq-2$ ) altered in at least one mutant compared to BRMS1 ${ }^{\mathrm{WT}}$. In order to analyze the significant interacting proteins in an unbiased manner, we used the dimension reduction method t-Stochastic Neighbor Embedding (tSNE), applied to Z-statistics obtained from QSPEC analysis [33]. This analysis resulted in four distinct clusters (Figure 2B, Supplementary Table 2). Further inspection identified BRMS1 as a member of cluster two, which contained the majority of the Sin3/HDAC members (Figure $\mathbf{2 C}$ ). This finding reinforces BRMS1 membership within Sin3/HDAC complexes while also refining the interactome by lack of association with SIN3B or SAP30L, which clustered differently (clusters 1 and 4, respectively) (Figure 2C). These findings are supported by cross-linking data (Figure $1 \mathrm{C}$ ), the combination of which could suggest that BRMS1 interacts more specifically with SIN3A than SIN3B complexes, but further investigation is needed to determine if that is the case.

\section{C-terminal mutants of BRMS1 disrupt protein interactions and molecular processes.}

Though significant that BRMS1 altered its binding patterns with all Sin3/HDAC members, many more proteins that exist outside of the Sin/HDAC complex had altered interactions with BRMS1 and its mutants. To understand what biological processes may be disrupted due to these altered interactions, all interacting proteins identified for each mutant were subjected to Reactome pathway analysis (Figure 3). Distinct differences in N-terminal (BRMS1-229) versus C-terminal (BRMS1 ${ }^{230-246}$ ) binding were observed. Many of the significant pathways represented in the analysis were not represented in BRMS1 ${ }^{1-229}$, e.g., mRNA processing, rRNA processing, and immune responses (Figure 3). Many of these same biological processes are disrupted by the C-terminus mutants (S237A, S237D, BRMS1 ${ }^{230-246}$ ), emphasizing the importance of the C-terminus in overall protein function and regulation of protein interactions. Importantly, many of the biological processes are tied to cancer-associated pathologies (i.e. mRNA splicing, immune response) and further study into the BRMS1 C-terminus role in these pathways could identify the molecular role of BRMS1 in metastasis. To identify binding partners distinctly affected by particular domains of BRMS1, all protein interaction changes for each mutant were considered. To ensure that putative interacting proteins were truly associated with BRMS1, an additional criterion was added, 
i.e., the interactor must have a high topological score (TopS) value in BRMS1 ${ }^{\mathrm{WT}}$, a value of which demonstrates a likelihood of binding ratio (Supplementary Table 3) [23]. Significant overlaps between the C-terminus associated mutants were observed. All three BRMS1 C-terminus mutants shared 64 proteins whose binding significantly changed compared to BRMS1 ${ }^{\mathrm{WT}}$ (Figure $4 \mathrm{~A}, \mathbf{4 B}$ ). These results are in sharp contrast to BRMS1 ${ }^{1-229}$, in which those same 64 proteins shared a more similar binding profile to BRMS1 $^{\text {WT }}$ (Figure 4B). Several of the 64 proteins are known to be promiscuous binders [i.e. Eukaryotic translation elongation factor 1 alpha 1 (EEF1A1), Heterogeneous nuclear ribonucleoprotein F (HNRNPF)]. To filter promiscuous binders from unique interactors within this dataset, these 64 proteins were subject to CRAPome analysis. $[23,35]$ Those found to be unique are colored in red in Figure 4B. Several of these proteins are associated with cancer, such as TRMT2A (TRNA Methyltransferase 2 Homolog A) and WWP1 (WW Domain Containing E3 Ubiquitin Protein Ligase 1). TRMT2A is a methyltransferase protein and the expression of this gene varies during the cell cycle, with aberrant expression being a possible biomarker in certain breast cancers [36]. Likewise, WWP1 is involved in breast mucinous carcinoma [37]. Two mutants, S237D and BRMS1 $1^{230-246}$, overlap with the most proteins, with 105 interactors (Figure 4A). These proteins were also subject to CRAPome analysis, which identified several proteins as unique. Two of these proteins are associated with metastasis, DNA Polymerase Epsilon Subunit 2 (POLE2) and Transcription Factor 2 (TCF2) [38, 39]. POLE2 is associated with circulating tumor cell clusters which, in turn, have been associated with an increased colonization success [38]. TCF2 plays a role in renal cell carcinoma patient progression [40]. In combination, these findings further support the role the C-terminus plays in protein specific binding, while emphasizing the importance of phosphorylation-status on that interaction, and potentially disease state.

To further characterize how changes in binding with these interactors may play a role in the development of certain pathologies, disease enrichment within Ingenuity Pathway Analysis (IPA) was completed (Figure 4C and Supplementary Table 4 (Worksheet 2)). This analysis included all interactor proteins with the C-terminus mutants (S237A, S237D, and BRMS130-246).

Several of the resulting pathologies are often associated with neoplasia, including cell cycle, cell death and survival, RNA damage repair, embryonic development, immunological disease, and infectious disease. The latter findings compliment previously published results in which re-expression of BRMS1 in metastatic MDA-MB-435 cells were highly enriched for upregulated immune response genes [41].

Two enriched diseases were of distinct interest. The first, tumor morphology, is of interest as previous studies showed that BRMS1 re-expression alters expression of the cytoskeletal proteins Focal Adhesion Kinase (FAK), Src, and Fascin [42-44]. Given that these proteins may ultimately change cancer 
cell morphology and motility [45], which are essential for cancer metastasis [1], the pathway was of special interest (Figure 4D). Additionally, cell death and survival pathways were as significantly enriched as tumor morphology. Previously BRMS1 has shown to be associated with inducing apoptosis in prostate and nonsmall cell lung cancers $[46,47]$. BRMS1 is also associated with HDAC1 and NF-KB, both of which are known play roles in regulating apoptosis [47].

Within these two enriched disease pathways, several proteins overlap, including BReast CAncer gene 1 (BRCA1), mechanistic target of rapamycin (mTOR), and Cyclin Dependent Kinase Inhibitor 2A (CDKN2A) (Figure 4D). Several of the proteins, shared or not, have been associated with many cancers (Figure 4D). Proteins that are associated with the C-terminus of BRMS1 strongly suggests that the state of S237 plays roles not only in how BRMS1 functions within the cellular environment, but a distinct role that may shape the metastatic potential within a cancer cell. This conclusion is consistent with previous reports that BRMS1 alters multiple steps in the metastatic cascade [42-44, 48].

\section{Location and phosphorylation status alter MDA-MB-231 migration patterns}

To begin assessing whether BRMS1 mutants exert different biological changes in cancer cells, all mutants were transduced into metastatic MDA-MB-231 breast carcinoma cells (Supplementary Figure 1). In vitro assays were performed to determine the impact of BRMS1 mutants on cell migration. Compared to both the parental MDA-MB-231s and vector only transduced cells, all mutants inhibited migration (Figures 5A, 5B). Curiously, BRMS1 ${ }^{230-246}$ reduced migration slightly more than BRMS1 ${ }^{1-229}$, but this comparison was not statistically significant. BRMS1 ${ }^{\mathrm{WT}}$ and S237D are significantly less migratory than BRMS1 ${ }^{1-229}$, while only S237D is less migratory than BRMS1 ${ }^{230-246}$. This finding may suggest that the capacity to exist within a phosphorylated state could decrease the migratory capacity. This observation is speculative, as neither S237A nor S237D have a statistically significantly different migratory capacity to BRMS1 ${ }^{\text {WT }}$, but the wound closure patterns are in opposite directions. Due to a lack of significant difference between S237A and S237D, the full impact of phosphorylation-status is merely speculative, and further in vitro and in vivo analyses need to be completed to confirm the entire impact on metastasis.

This study investigates BRMS1's C-terminus role in protein function, and specifically its regulation of BRMS1 protein bindings. We demonstrate that the C-terminus plays a significant role in regulating its binding partners and validates the importance that S237 phosphorylation plays in regulating these partners. Though further studies need to be completed to determine how BRMS1 suppresses metastasis, this study acutely demonstrates that the C-terminus regulates BRMS1 binding functions begins to explain 
how the C-terminus of BRMS impacts metastasis suppressor function. Future studies will focus on elucidating this regulation as it may be key to unlocking how BRMS1 functions to suppress metastasis.

\section{Materials and Methods:}

\section{Cloning of N-terminally Halo-tagged versions of BRMS1 in pCDNA5/FRT.}

The BRMS1 primers listed in Supplementary Data were used to amplify a sequence coding for BRMS1 isoform 1 (NP_056214), or for mutant versions of BRMS1 as indicated in the figure legends, using a previously reported BRMS1 construct as a template [20]. A short synthetic duplex DNA oligonucleotide (described in Supplementary Data) was used to clone a short fragment of the C terminus of BRMS1 (amino acids 230-246). DNA fragments were digested with Sgfl and Pmel and inserted between Sgfl and Pmel sites in pcDNA5FRT-Halo [12].

\section{Affinity purification of BRMS1 for proteomic analysis.}

HEK293T cells $\left(1 \times 10^{7}\right)$ were cultured into a $15 \mathrm{~cm}$ tissue culture plates for 24 hours then Halo-BRMS1 constructs were transfected using Lipofectamine LTX (Thermo Fisher Scientific). After 48 hours cells were harvested and washed twice with ice-cold PBS. Cells were then resuspended in mammalian cell lysis buffer (Promega) (50 mM Tris· $\mathrm{HCl}$ (pH 7.5), $150 \mathrm{mM} \mathrm{NaCl}, 1 \%$ Triton $^{\circledR} \mathrm{X}-100,0.1 \%$ sodium deoxycholate, $0.1 \mathrm{mM}$ benzamidine $\mathrm{HCl}, 55 \mu \mathrm{M}$ phenanthroline, $1 \mathrm{mM}$ PMSF, $10 \mu \mathrm{M}$ bestatin, $5 \mu \mathrm{M}$ pepstatin $\mathrm{A}$, and 20 $\mu \mathrm{M}$ leupeptin) followed by centrifugation at $21,000 \times \mathrm{g}$ for $10 \mathrm{~min}$ at $4{ }^{\circ} \mathrm{C}$. To remove insoluble materials, cell extracts were diluted with $700 \mu \mathrm{l}$ of TBS (50 mM Tris. $\mathrm{HCl} \mathrm{pH} \mathrm{7.4,} 137 \mathrm{mM} \mathrm{NaCl}, 2.7 \mathrm{mM} \mathrm{KCl}$ ) and centrifuged at $21,000 \times g$ for $10 \mathrm{~min}$ at $4{ }^{\circ} \mathrm{C}$. Next, cell extracts were incubated overnight at $4{ }^{\circ} \mathrm{C}$ with magnetic beads (Magne ${ }^{\mathrm{TM}}$ HaloTag ${ }^{\circledR}$ slurry). Before elution, magnetic beads were washed four times with wash buffer (50 mM Tris- $\mathrm{HCl} \mathrm{pH} \mathrm{7.4,} 137 \mathrm{mM} \mathrm{NaCl}, 2.7 \mathrm{mM} \mathrm{KCl}$, and 0.05\% Nonidet ${ }^{\circledR}$ P40). Proteins bound to magnetic beads were eluted for 2 hours at room temperature using elution buffer containing $50 \mathrm{mM}$ Tris- $\mathrm{HCl}$ pH 8.0, $0.5 \mathrm{mM}$ EDTA, $0.005 \mathrm{mM}$ DTT, and 2 Units AcTEV TM Protease (Thermo Fisher Scientific). The eluate was further purified by passing through a Micro Bio-Spin column (Bio-Rad, Hercules, CA) to remove residual beads prior to proteomic analyses.

\section{MudPIT analysis for BRMS1 and BRMS1 mutant associated proteins.}

MudPIT analysis for protein identification was previously reported in detail by Banks et al. [12]. Briefly, trichloroacetic acid (TCA) precipitated proteins were proteolytically digested with endoproteinase Lys-C and trypsin digestion, respectively. Digested peptides were injected directly into a linear ion trap (LTQ) mass spectrometer using 10-step MudPIT separation approach, then the yielded spectra were collected 
and identified. Spectra were analyzed using the ProLuCID and DTASelect algorithms. Contrast and NSAF7 software were used, respectively, to rank putative affinity purified proteins according to their distributed normalized spectral abundance values (dNSAF). QSPEC was used to identify enriched proteins in the experimental samples [12].

\section{Cross-linking Analysis}

Cross-linking data were utilized from publicly available data published by Adams et. al 2020 [21]. The BRMS1 cross-links were visualized within the xiView platform [22].

\section{Topological Scoring}

Topological Scoring was completed for all proteins as previously described [23, 24]. Briefly, proteins with significant QSPEC scores were input into the TopS Shiny Application (available at https://github.com/WashburnLab/Topological-score-TopS). This application utilizes the average spectral counts of each bait across all baits to calculate the TopS score, which indicates a likelihood ratio of binding.

\section{Protein Structure Predictions}

BRMS1 protein structure was predicted based upon its amino acid sequence retrieved from NCBI using I-TASSER $[25,26]$. Briefly, this program functions predicts, with high accuracy, protein structure based upon primary amino acid sequences by comparing the inputted sequence to known BLAST sequences. This comparison is then used to identify potential protein relatives, followed by threading, in which the LOMETS database utilizes the PDB database to generate alignments. Alignments with sufficient Z-Scores are then excised and assembled. Predicted structures are then subjected to iterative Monte Carlo simulations within a variety of conditions (temperature change simulations, etc.). Predictions with the lowest energy state are selected to be the predictive protein structures before comparing against knowns in the PDB database for structure, ligand binding, and biological function.

\section{Protein Stability Predictions}

The primary sequence of BRMS1 was mutated at S237 to either Aspartic Acid (D) or Alanine (A). These mutants were subject to multiple predictive software applications. MuPro [27] utilizes multiple machine learning techniques to predict the impact a point mutation has on protein stability. I-Mutant [28] is a support vector machine-based tool that functions by utilizing neural networks to predict protein stability changes based upon point mutations. Folding RaCe [29] predictive software utilizes a 790 single point mutant knowledge-based prediction of protein folding rates with a multiple linear regression model approach to predict the impact of single site mutations on folding rates. DynaMut [30] combines normal mode analysis which approximates system dynamics and motions with the mutational analysis, such that the prediction is based upon the impact that the mutation has on overall protein dynamics. DIM-Pred [31] 
predicts order or disorder of a protein or a protein region, accounting for alterations in amino acid properties, neighboring amino acids residues, and substation matrices.

\section{Data Analysis}

Three biological replicates were performed for each bait protein (i.e. BRMS1 wild-type and four mutants). The distributed normalized spectral abundance factor (dNSAF) [32] was used to quantify the prey proteins in each bait AP-MS. To eliminate potential nonspecific proteins, three negative controls were analyzed from cells expressing the Halo tag alone. A total of 15 purifications were completed and 5085 prey proteins identified (Supplementary File 1). First, wild-type data were compared against the negative control dataset to ensure that non-specific proteins were not included in the analysis (Supplementary Table S1). Second, QSPEC [33] was used on this filtered protein list to calculate Z-statistics between spectral counts measured in wild-type and mutants and determine significant changes in protein levels between these two datasets (Supplementary Table 2). We retained only proteins that had a significant QSPEC Z-statistics of -2 or less in at least one of the mutants. The final group of 1175 proteins that passed these criteria comprised the subunits of the Sin3/HDAC complex and proteins outside the complex.

\section{t-SNE}

To spatially map all significant 1175 proteins, we first applied a T-Distributed Stochastic Neighbor Embedding(t-SNE), a nonlinear visualization of the data followed by a k-means clustering on the two vectors generated from the t-SNE (Supplementary Table 2). The number of clusters used for the k-means was 4 . The plot visualization was generated within the R packages stats, cluster, gplots, and ggplot2.

\section{Pathway analysis.}

In order to determine the biological enrichment of differentially expressed proteins in the mutants, we subjected 1175 proteins to Reactome pathway analysis. Enrichment was completed within the R environment and several packages such as clusterProfiler, ReactomePA, DOSE and enrichplot were used.

\section{Protein Functional Analysis}

Significant proteins were subjected to multiple analyses. Disease enrichment was completed within Ingenuity Pathway Analysis (IPA, http://www.ingenuity.com, Release date: December 2016). Visualization for overlapping significant proteins was completed in Venny 2.1 (https://bioinfogp.cnb.csic.es/tools/venny/). Heatmaps were generated within ClustVis (https://biit.cs.ut.ee/clustvis/).

\section{Cell Culture}

MDA-MB-231 cells were cultured in 5\% FBS, 0.5 mM NEAA 0.5 mM NEAA + DMEM/Sodium Bicarbonate $(2.438 \mathrm{~g} / \mathrm{L})$ cell culture medium (Thermo Fisher Scientific). Overexpression cells were first transduced with 
pENTR followed by pLENTI expression within 293FT cells according to manufacturer's instructions (Invitrogen). Clones were selected with blasticidin. Each construct contained a single or 3x-Flag epitope tag. Expression was quantified using validated BRMS1 1a5.7 antibody (as described previously [34]), FLAGM2 antibody (Sigma A8592-IMG), or qPCR using the following primers:

F: 5'GATCCATGGACTACAAAGACCATGACGGTGATTATAAAGATCATGACATCGATTACAAGGATGACGATG ACAAGAAGGCTAGGGCAGCTGTGTCCCCTCAGAAGAGAAAATCGGATGGACCTTGATGATGAC -3', $\mathrm{R}:$ $5^{\prime}$

TCGAGTCATCATCAAGGTCCATCCGATTTTCTCTTCTGAGGGGACACAGCTGCCCTAGCCTTCTGTCATCG TCATCCTTGTAATCGATGTCATGATCTTTATAATCACCGTCATGGTCTTTGTAGTCCATG -3'

Predicted amino acid sequences for each construct are:

BRMS1 $^{\text {WT }}$

DYKDDDDKMPVQPPSKDTEEMEAEGDSAAEMNGEEEESEEERSGSQTESEEESSEMDDEDYERRRSECVSE MLDLEKQFSELKEKLFRERLSQLRLRLEEVGAERAPEYTEPLGGLQRSLKIRIQVAGIYKGFCLDVIRNKYECELQ GAKQHLESEKLLLYDTLQGELQERIQRLEEDRQSLDLSSEWWDDKLHARGSSRSWDSLPPSKRKKAPLVSGPYI VYMLQEIDILEDWTAIKKARAAVSPQKRKSDGP

BRMS1 $^{1-229}$

DYKDDDDKMPVQPPSKDTEEMEAEGDSAAEMNGEEEESEEERSGSQTESEEESSEMDDEDYERRRSECVSE MLDLEKQFSELKEKLFRERLSQLRLRLEEVGAERAPEYTEPLGGLQRSLKIRIQVAGIYKGFCLDVIRNKYECELQ GAKQHLESEKLLLYDTLQGELQERIQRLEEDRQSLDLSSEWWDDKLHARGSSRSWDSLPPSKRKKAPLVSGPYI VYMLQEIDILEDWTAI

S237A

DYKDDDDKMPVQPPSKDTEEMEAEGDSAAEMNGEEEESEEERSGSQTESEEESSEMDDEDYERRRSECVSE MLDLEKQFSELKEKLFRERLSQLRLRLEEVGAERAPEYTEPLGGLQRSLKIRIQVAGIYKGFCLDVIRNKYECELQ GAKQHLESEKLLLYDTLQGELQERIQRLEEDRQSLDLSSEWWDDKLHARGSSRSWDSLPPSKRKKAPLVSGPYI VYMLQEIDILEDWTAIKKARAAVAPQKRKSDGP 
DYKDDDDKMPVQPPSKDTEEMEAEGDSAAEMNGEEEESEEERSGSQTESEEESSEMDDEDYERRRSECVSE MLDLEKQFSELKEKLFRERLSQLRLRLEEVGAERAPEYTEPLGGLQRSLKIRIQVAGIYKGFCLDVIRNKYECELQ GAKQHLESEKLLLYDTLQGELQERIQRLEEDRQSLDLSSEWWDDKLHARGSSRSWDSLPPSKRKKAPLVSGPYI VYMLQEIDILEDWTAIKKARAAVDPQKRKSDGP

BRMS1 $^{230-246}$

\section{MDYKDHDGDYKDHDIDYKDDDDKKARAAVSPQKRKSDGP}

\section{Migration Assay}

Cells were cultured overnight in 5\% FBS, 0.5 mM NEAA + DMEM/Sodium Bicarbonate $(2.438 \mathrm{~g} / \mathrm{L})$ media in 6-well plates (Thermo Fisher). After 48 hours cells were scratched, washed, and media replaced with serum-free DMEM/Sodium Bicarbonate ( $2.438 \mathrm{~g} / \mathrm{L}$ ) (Thermo Fisher). Cells were imaged at $\mathrm{t}=18$ to $24 \mathrm{hrs}$. Image J (https://imagej.nih.gov/ij/) was utilized for analysis, followed by statistical analysis in R ('FAS' package), in which a Kruskal Wallis test followed by a Dunn's adjustment was completed (https://www.Rproject.org/).

\section{Visualizations}

R packages Gplots, GGplots2, and RColor Brewer were utilized for image analysis of data.

\section{Acknowledgements}

Funding - This work was supported by the Stowers Institute for Medical Research (MPW, MES, MSM, CAB, MKA); National Foundation for General Medicine [GM112639, F32GM122215 (MPW)]; National Foundation for Cancer Research (DRW), USPHS National Cancer Institute [CA134981, CA168524 (DRW)]; American Cancer Society [PF-16-227-01-CSM (CAM)]; Susan G. Komen for the Cure [SAC110037 (DRW)]; METAvivor Research and Support, Inc. (DRW); The K-INBRE Bioinformatics Core supported in part by the National Institute of General Medical Science [P20-GM103418 (RCZ]; the KU Cancer Center Biostatistics and Informatics Shared Resource supported by the National Cancer Institute Cancer Center [P30CA168524 (DK, DRW)]; and the Kansas Institute for Precision Medicine COBRE supported by the National Institute of General Medical Science [P20-GM130423 (DCK)].

Data Availability Statement - The mass spectrometry datasets generated for this study are available from the Massive data repository (https://massive.ucsd.edu) using the identifiers listed in Supplementary Table 5. For data generated at the Stowers Institute, links to the original data underlying this manuscript can be 


accessed from the Stowers Original Data Repository at
http://www.stowers.org/research/publications/libpb-1585

COI

The authors declare no conflicts of interest.

\section{Author Contributions}

RCZ: Conceptualization; data curation; formal analysis; writing-original draft; writing-editing

MES: Formal analysis; writing-original draft; writing-editing

CAM: Conceptualization; funding acquisition; methodology; writing-original draft; writing-editing

MSM: Data curation; formal analysis; methodology; writing-editing

CAB: Data curation; formal analysis; methodology; writing-editing

MKA: Data curation; formal analysis; methodology; writing-editing

DCK: Formal analysis; writing-editing

MPW: Conceptualization; supervision; funding acquisition; methodology; project administration; writing-editing

DRW: Conceptualization; supervision; funding acquisition; methodology; project administration; writing-editing

\section{ORCID}

$\begin{array}{ll}\text { RCZ } & 0000-0001-8611-2545 \\ \text { MES } & 0000-0002-3987-7569 \\ \text { CAM } & 0000-0003-1356-828 X \\ \text { MSM } & 0000-0002-5901-5181 \\ \text { CAB } & 0000-0002-0404-2940 \\ \text { MKA } & 0000-0002-1078-9514 \\ \text { DCK } & 0000-0002-0598-0146 \\ \text { MPW } & 0000-0001-7568-2585 \\ \text { DRW } & 0000-0002-1951-4947\end{array}$

\section{References}

[1] D.R. Welch, D.R. Hurst, Defining the Hallmarks of Metastasis, Cancer Res., 79 (2019) 30113027. 
[2] Y. Liu, M.W. Mayo, A. Xiao, E.H. Hall, E.B. Amin, K. Kadota, P.S. Adusumilli, D.R. Jones, Loss of BRMS1 Promotes a Mesenchymal Phenotype through NF-kappaB-Dependent Regulation of Twist1, Mol. Cell Biol, (2014).

[3] P.S. Steeg, T. Ouatas, D. Halverson, D. Palmieri, M. Salerno, Metastasis suppressor genes: Basic biology and potential clinical use, Clinical Breast Cancer, 5 (2003) 51-62.

[4] W. Liu, C.J. Vivian, A.E. Brinker, K.R. Hampton, E. Lianidou, D.R. Welch, Microenvironmental Influences on Metastasis Suppressor Expression and Function during a Metastatic Cell's Journey, Cancer Microenviron., 7 (2014) 117-131.

[5] M.J. Seraj, R.S. Samant, M.F. Verderame, D.R. Welch, Functional evidence for a novel human breast carcinoma metastasis suppressor, BRMS1, encoded at chromosome 11q13, Cancer Research, 60 (2000) 2764-2769.

[6] M.J. Seraj, M.A. Harding, J.J. Gildea, D.R. Welch, D. Theodorescu, The relationship of BRMS1 and RhoGDI2 gene expression to metastatic potential in lineage related human bladder cancer cell lines, Clin Exp Metastasis, 18 (2000) 519-525.

[7] L.A. Shevde, R.S. Samant, D.R. Welch, Suppression of human melanoma metastasis by breast metastasis suppressor [BRMS1], PNAS, 42 (2001) 646.

[8] P.R. Bucciarelli, K.S. Tan, N.P. Chudgar, W. Brandt, J. Montecalvo, T. Eguchi, Y. Liu, R. Aly, W.D. Travis, P.S. Adusumilli, D.R. Jones, BRMS1 Expression in Surgically Resected Lung Adenocarcinoma Predicts Future Metastases and Is Associated with a Poor Prognosis, J. Thorac. Oncol., 13 (2018) 73-84.

[9] J. Li, Y. Cheng, D. Tai, M. Martinka, D.R. Welch, G. Li, Prognostic significance of BRMS1 expression in human melanoma and its role in tumor angiogenesis, Oncogene, 30 (2011) 896906.

[10] Z. Zhang, H. Yamashita, T. Toyama, Y. Yamamoto, T. Kawasoe, H. Iwase, Reduced expression of the breast cancer metastasis suppressor $1 \mathrm{mRNA}$ is correlated with poor progress in breast cancer, Clin Cancer Res, 12 (2006) 6410-6414.

[11] W.J. Meehan, R.S. Samant, J.E. Hopper, M.J. Carrozza, L.A. Shevde, J.L. Workman, K.A. Eckert, M.F. Verderame, D.R. Welch, Breast cancer metastasis suppressor 1 (BRMS1) forms complexes with retinoblastoma-binding protein 1 (RBP1) and the $\mathrm{mSin} 3$ histone deacetylase complex and represses transcription, J. Biol. Chem., 279 (2004) 1562-1569.

[12] C.A.S. Banks, Y. Zhang, S. Miah, Y. Hao, M.K. Adams, Z. Wen, J.L. Thornton, L. Florens, M.P. Washburn, Integrative Modeling of a Sin3/HDAC Complex Sub-structure, Cell Rep., 31 (2020) 107516.

[13] M.E. Sardiu, K.T. Smith, B.D. Groppe, J.M. Gilmore, A. Saraf, R. Egidy, A. Peak, C.W. Seidel, L. Florens, J.L. Workman, M.P. Washburn, Suberoylanilide hydroxamic acid (SAHA)-induced dynamics of a human histone deacetylase protein interaction network, Mol Cell Proteomics, 13 (2014) 3114-3125.

[14] Y. Liu, M.W. Mayo, A.S. Nagji, E.H. Hall, L.S. Shock, A. Xiao, E.B. Stelow, D.R. Jones, BRMS1 suppresses lung cancer metastases through an E3 ligase function on histone acetyltransferase p300, Cancer Res., 73 (2013) 1308-1317.

[15] D. Pantoja-Uceda, J.L. Neira, L.M. Contreras, C.A. Manton, D.R. Welch, B. Rizzuti, The isolated C-terminal nuclear localization sequence of the breast cancer metastasis suppressor 1 is disordered, Arch Biochem Biophys, 664 (2019) 95-101. 
[16] M. Spinola-Amilibia, J. Rivera, M. Ortiz-Lombardia, A. Romero, J.L. Neira, J. Bravo, The structure of BRMS1 nuclear export signal and SNX6 interacting region reveals a hexamer formed by antiparallel coiled coils, J Mol Biol, 411 (2011) 1114-1127.

[17] J. Rivera, D. Megias, J. Bravo, Sorting nexin 6 interacts with breast cancer metastasis suppressor-1 and promotes transcriptional repression, J Cell Biochem, 111 (2010) 1464-1472. [18] D.R. Hurst, Y. Xie, J.W. Thomas, J. Liu, M.D. Edmonds, M.D. Stewart, D.R. Welch, The Cterminal putative nuclear localization sequence of breast cancer metastasis suppressor 1 , BRMS1, is necessary for metastasis suppression, PLoS One, 8 (2013) e55966.

[19] S.N. Roesley, R. Suryadinata, E. Morrish, A.R. Tan, S.M. Issa, J.S. Oakhill, O. Bernard, D.R. Welch, B. Sarcevic, Cyclin-dependent kinase-mediated phosphorylation of breast cancer metastasis suppressor 1 (BRMS1) affects cell migration, Cell Cycle, 15 (2016) 137-151.

[20] M.K. Adams, C.A.S. Banks, J.L. Thornton, C.G. Kempf, Y. Zhang, S. Miah, Y. Hao, M.E. Sardiu, M. Killer, G.L. Hattem, A. Murray, M.L. Katt, L. Florens, M.P. Washburn, Differential Complex Formation via Paralogs in the Human Sin3 Protein Interaction Network, Mol Cell Proteomics, 19 (2020) 1468-1484.

[21] M.K. Adams, C.A.S. Banks, J.L. Thornton, M.E. Sardiu, M. Killer, C.G. Kempf, L. Florens, M.P. Washburn, Differential complex formation via paralogs in the human Sin3 protein interaction network, bioRxiv, (2019) 830828.

[22] M. Graham, C. Combe, L. Kolbowski, J. Rappsilber, xiView: A common platform for the downstream analysis of Crosslinking Mass Spectrometry data, bioRxiv, (2019) 561829. [23] M.E. Sardiu, J.M. Gilmore, B.D. Groppe, A. Dutta, L. Florens, M.P. Washburn, Topological scoring of protein interaction networks, Nat Commun, 10 (2019) 1118.

[24] M.E. Sardiu, L. Florens, M.P. Washburn, Generating topological protein interaction scores and data visualization with TopS, Methods, 184 (2020) 13-18.

[25] Y. Zhang, I-TASSER server for protein 3D structure prediction, BMC Bioinformatics, 9 (2008) 40.

[26] A. Roy, A. Kucukural, Y. Zhang, I-TASSER: a unified platform for automated protein structure and function prediction, Nat Protoc, 5 (2010) 725-738.

[27] J. Cheng, A. Randall, P. Baldi, Prediction of protein stability changes for single-site mutations using support vector machines, Proteins, 62 (2006) 1125-1132.

[28] E. Capriotti, P. Fariselli, R. Casadio, I-Mutant2.0: predicting stability changes upon mutation from the protein sequence or structure, Nucleic Acids Res, 33 (2005) W306-310.

[29] P. Chaudhary, A.N. Naganathan, M.M. Gromiha, Folding RaCe: a robust method for predicting changes in protein folding rates upon point mutations, Bioinformatics, 31 (2015) 2091-2097.

[30] C.H. Rodrigues, D.E. Pires, D.B. Ascher, DynaMut: predicting the impact of mutations on protein conformation, flexibility and stability, Nucleic Acids Res, 46 (2018) W350-W355. [31] P. Anoosha, R. Sakthivel, M.M. Gromiha, Prediction of protein disorder on amino acid substitutions, Anal Biochem, 491 (2015) 18-22.

[32] Y. Zhang, Z. Wen, M.P. Washburn, L. Florens, Refinements to label free proteome quantitation: how to deal with peptides shared by multiple proteins, Anal Chem, 82 (2010) 2272-2281. 
[33] H. Choi, S. Kim, D. Fermin, C.C. Tsou, A.I. Nesvizhskii, QPROT: Statistical method for testing differential expression using protein-level intensity data in label-free quantitative proteomics, J Proteomics, 129 (2015) 121-126.

[34] D.R. Hurst, Y. Xie, K.S. Vaidya, A. Mehta, B.P. Moore, M.A. Accavitti-Loper, R.S. Samant, R. Saxena, A.C. Silveira, D.R. Welch, Alterations of BRMS1-ARID4A interaction modify gene expression but still suppress metastasis in human breast cancer cells, J. Biol. Chem., 283 (2008) 7438-7444.

[35] D. Mellacheruvu, Z. Wright, A.L. Couzens, J.P. Lambert, N.A. St-Denis, T. Li, Y.V. Miteva, S. Hauri, M.E. Sardiu, T.Y. Low, V.A. Halim, R.D. Bagshaw, N.C. Hubner, A. Al-Hakim, A. Bouchard, D. Faubert, D. Fermin, W.H. Dunham, M. Goudreault, Z.Y. Lin, B.G. Badillo, T. Pawson, D. Durocher, B. Coulombe, R. Aebersold, G. Superti-Furga, J. Colinge, A.J. Heck, H. Choi, M. Gstaiger, S. Mohammed, I.M. Cristea, K.L. Bennett, M.P. Washburn, B. Raught, R.M. Ewing, A.C. Gingras, A.I. Nesvizhskii, The CRAPome: a contaminant repository for affinity purification-mass spectrometry data, Nat Methods, 10 (2013) 730-736.

[36] D.G. Hicks, B.R. Janarthanan, R. Vardarajan, S.A. Kulkarni, T. Khoury, D. Dim, G.T. Budd, B.J. Yoder, R. Tubbs, M.T. Schreeder, N.C. Estopinal, R.A. Beck, Y. Wang, B.Z. Ring, R.S. Seitz, D.T. Ross, The expression of TRMT2A, a novel cell cycle regulated protein, identifies a subset of breast cancer patients with HER2 over-expression that are at an increased risk of recurrence, BMC Cancer, 10 (2010) 108.

[37] C. Chen, Z. Zhou, J.S. Ross, W. Zhou, J.T. Dong, The amplified WWP1 gene is a potential molecular target in breast cancer, Int. J. Cancer, 121 (2007) 80-87.

[38] S. Gkountela, F. Castro-Giner, B.M. Szczerba, M. Vetter, J. Landin, R. Scherrer, I. Krol, M.C. Scheidmann, C. Beisel, C.U. Stirnimann, C. Kurzeder, V. Heinzelmann-Schwarz, C. Rochlitz, W.P. Weber, N. Aceto, Circulating Tumor Cell Clustering Shapes DNA Methylation to Enable Metastasis Seeding, Cell, 176 (2019) 98-112.e114.

[39] A. Buchner, M. Castro, A. Hennig, T. Popp, G. Assmann, C.G. Stief, W. Zimmermann, Downregulation of HNF-1B in renal cell carcinoma is associated with tumor progression and poor prognosis, Urology, 76 (2010) 507.e506-511.

[40] T.M. Frayling, H. Colhoun, J.C. Florez, A genetic link between type 2 diabetes and prostate cancer, Diabetologia, 51 (2008) 1757-1760.

[41] P.J. Champine, J. Michaelson, B.C. Weimer, D.R. Welch, D.B. DeWald, Microarray analysis reveals potential mechanisms of BRMS1-mediated metastasis suppression, Clin Exp Metastasis, 24 (2007) 551-565.

[42] P. Mei, J. Bai, M. Shi, Q. Liu, Z. Li, Y. Fan, J. Zheng, BRMS1 suppresses glioma progression by regulating invasion, migration and adhesion of glioma cells, PLoS One, 9 (2014) e98544. [43] Y.B. Khotskaya, B.H. Beck, D.R. Hurst, Z. Han, W. Xia, M.C. Hung, D.R. Welch, Expression of metastasis suppressor BRMS1 in breast cancer cells results in a marked delay in cellular adhesion to matrix, Mol Carcinog, 53 (2014) 1011-1026.

[44] L.A. Shevde, R.S. Samant, S.F. Goldberg, T. Sikaneta, A. Alessandrini, H.J. Donahue, D.T. Mauger, D.R. Welch, Suppression of human melanoma metastasis by the metastasis suppressor gene, BRMS1, Exp Cell Res, 273 (2002) 229-239.

[45] P. Friedl, Y. Hegerfeldt, M. Tusch, Collective cell migration in morphogenesis and cancer, Int J Dev Biol, 48 (2004) 441-449. 
[46] H.M. Zhang, Q.D. Qiao, H.F. Xie, J.X. Wei, Breast cancer metastasis suppressor 1 (BRMS1) suppresses prostate cancer progression by inducing apoptosis and regulating invasion, Eur Rev Med Pharmacol Sci, 21 (2017) 68-75.

[47] Y. Liu, P.W. Smith, D.R. Jones, Breast cancer metastasis suppressor 1 functions as a corepressor by enhancing histone deacetylase 1-mediated deacetylation of RelA/p65 and promoting apoptosis, Mol Cell Biol, 26 (2006) 8683-8696.

[48] P.W. Smith, Y. Liu, S.A. Siefert, C.A. Moskaluk, G.R. Petroni, D.R. Jones, Breast cancer metastasis suppressor 1 (BRMS1) suppresses metastasis and correlates with improved patient survival in non-small cell lung cancer, Cancer Lett., 276 (2009) 196-203. 


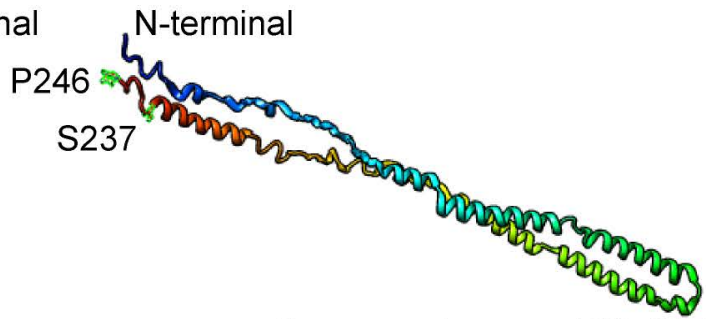

Green region: aa 100-155

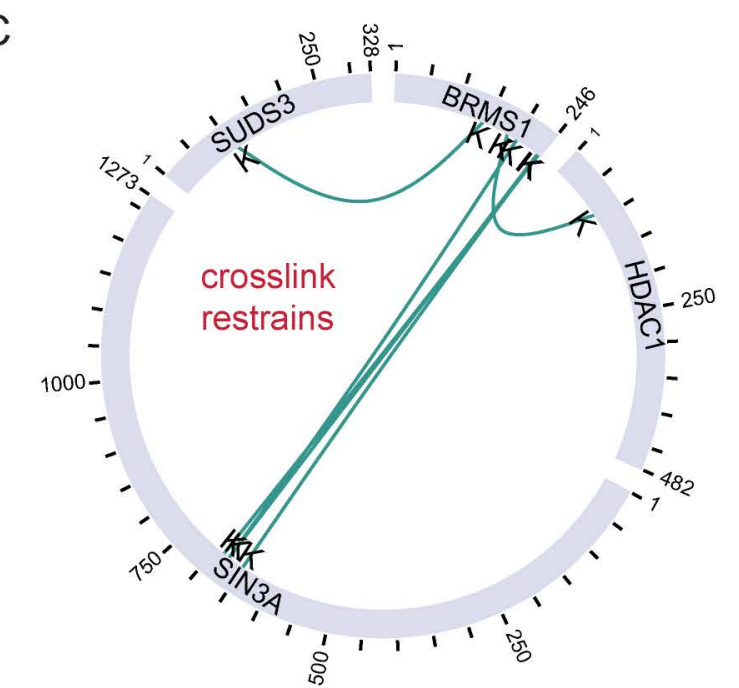

Figure 1. Structural analysis of BRMS1 demonstrates regions associated with protein-protein binding. Secondary structure prediction of the BRMS1 proteins by I-TASSER (A), followed by the BRMS1 predicted 3D model (B). The structure generated demonstrates that the $\mathrm{C}$-terminal region consists of helical, coiled, and strand regions. (C) Crosslink map for the BRMS1 protein, data is extracted from SIN3A XL-MS experiments from Adams et al (36). 


\section{BRMS1wT}

BRMS1 1-229

229

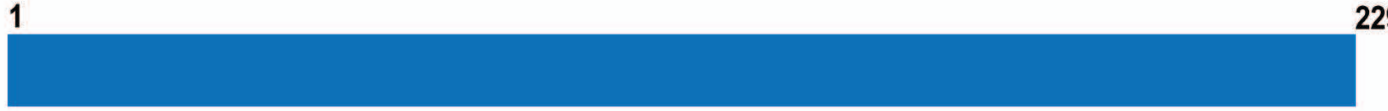

1

$237 \quad 246$

S237A

1

$237 \quad 246$

S237D

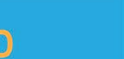

BRMS1230-246

230

246
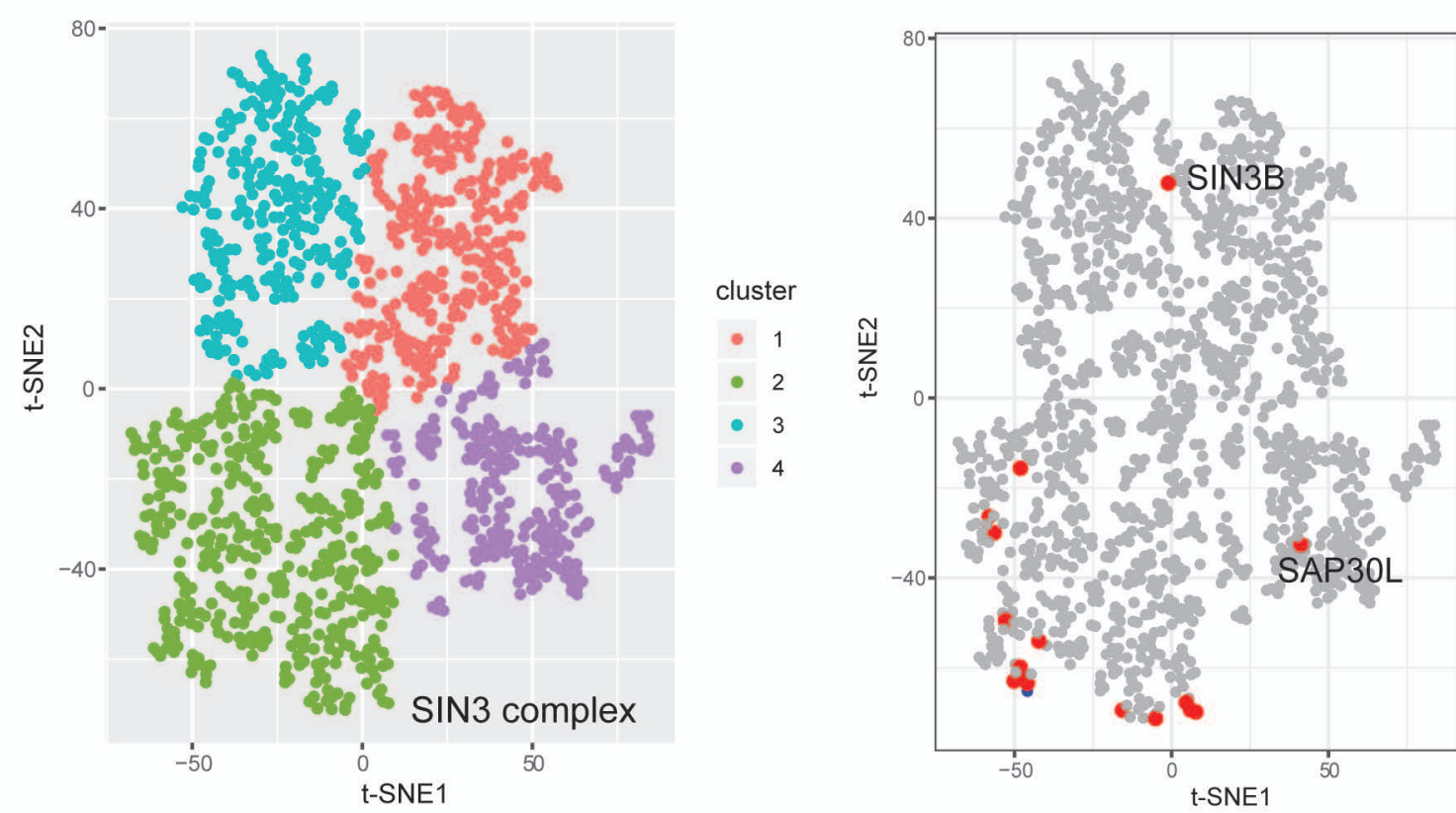

label

- protein interactions

outside the SIN3/HDAC complex

- subunits of the Sin3/HDAC complex

- BRMS1

Figure 2. Cluster analysis of mutants associates BRMS1 with selected members of the Sin3/HDAC

complex. (A) Mass spectrometry was completed on four BRMS1 mutants with full-length BRMS1 (BRMS1WT), BRMS1 aa 1-229 (BRMS11-229), BRMS1S237A, BRMS1S237D, and BRMS1 aa 230-246 (BRMS1230-246).

(B) t-SNE analysis was implemented for the analysis of the mutant data. Four clusters are generated by t-SNE

(B). The network was colored by the cluster numbers in Figure $2 \mathrm{C}$. In the case of Figure $2 \mathrm{C}$, components of the Sin3/HDAC (in red) and BRMS1(in blue) protein are highlighted. 
bioRxiv preprint doi: https://doi.org/10.1101/2021.02.04.429764; this version posted February 4, 2021. The copyright holder for this preprint (which was not certified by peer review) is the author/funder. All rights reserved. No reuse allowed without permission.

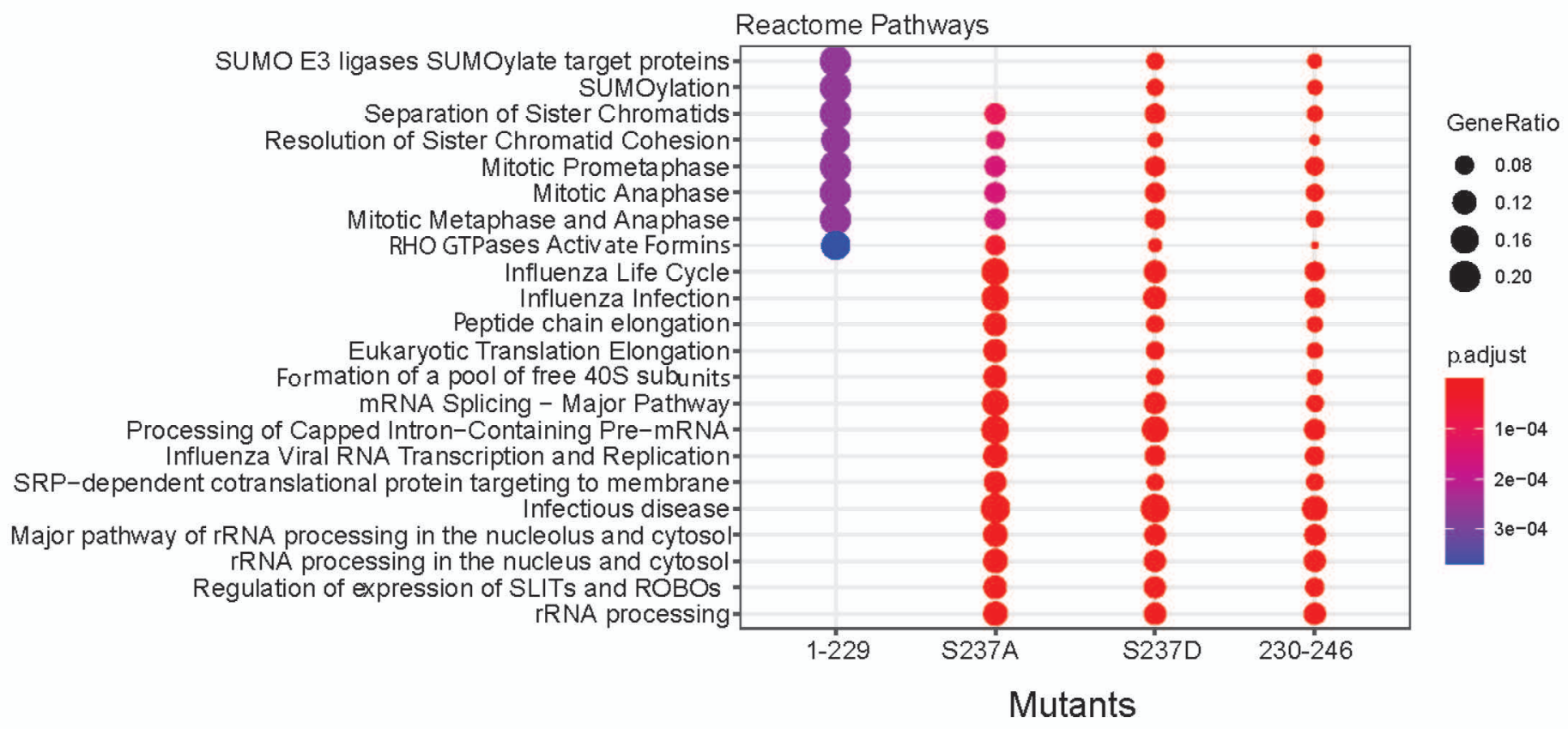

Figure 3. Biological pathway analysis associates BRMS1 mutants with specific molecular processes. The total number of 1175 proteins were subject to the Reactome pathway analysis. Top significant biological pathways with a p-value $<0.01$ corresponding to the four mutants are represented in this figure. 

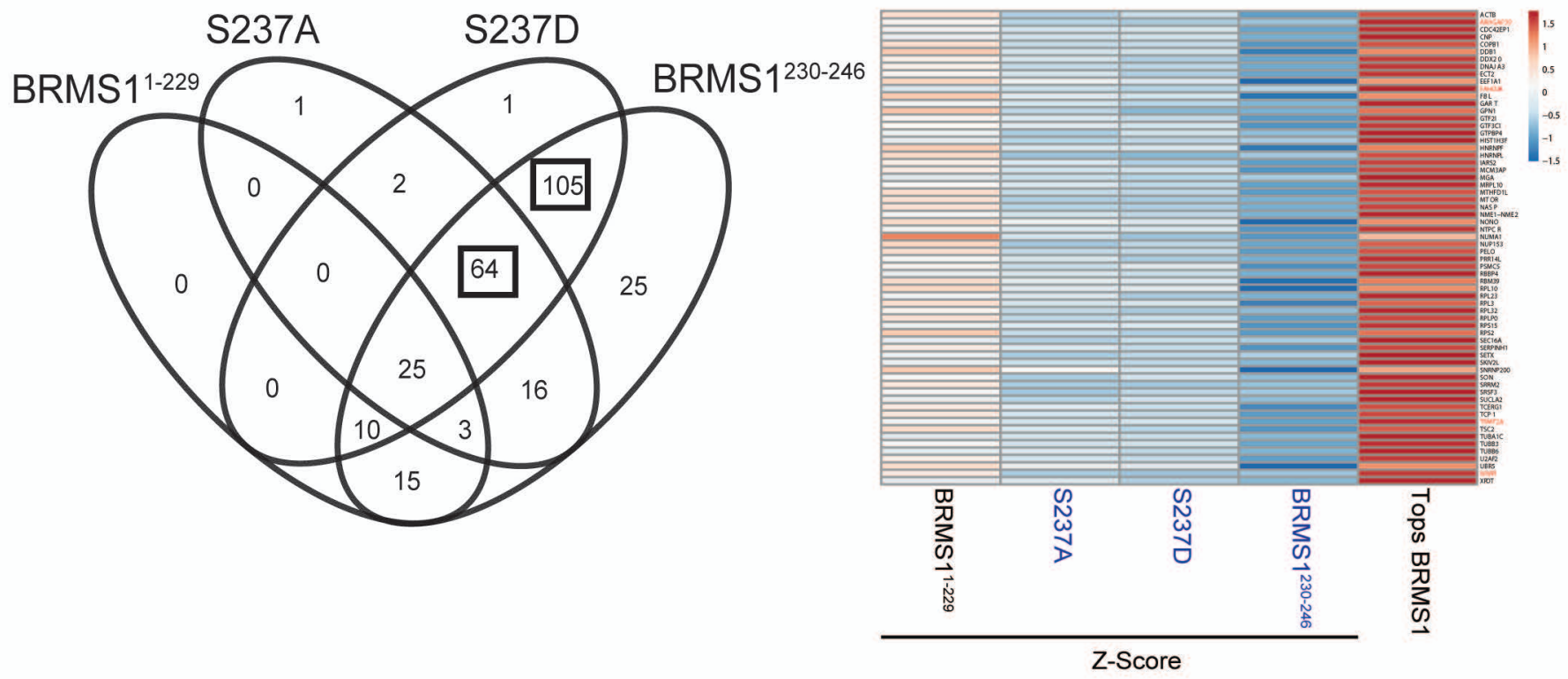

C

$\mathrm{D}$
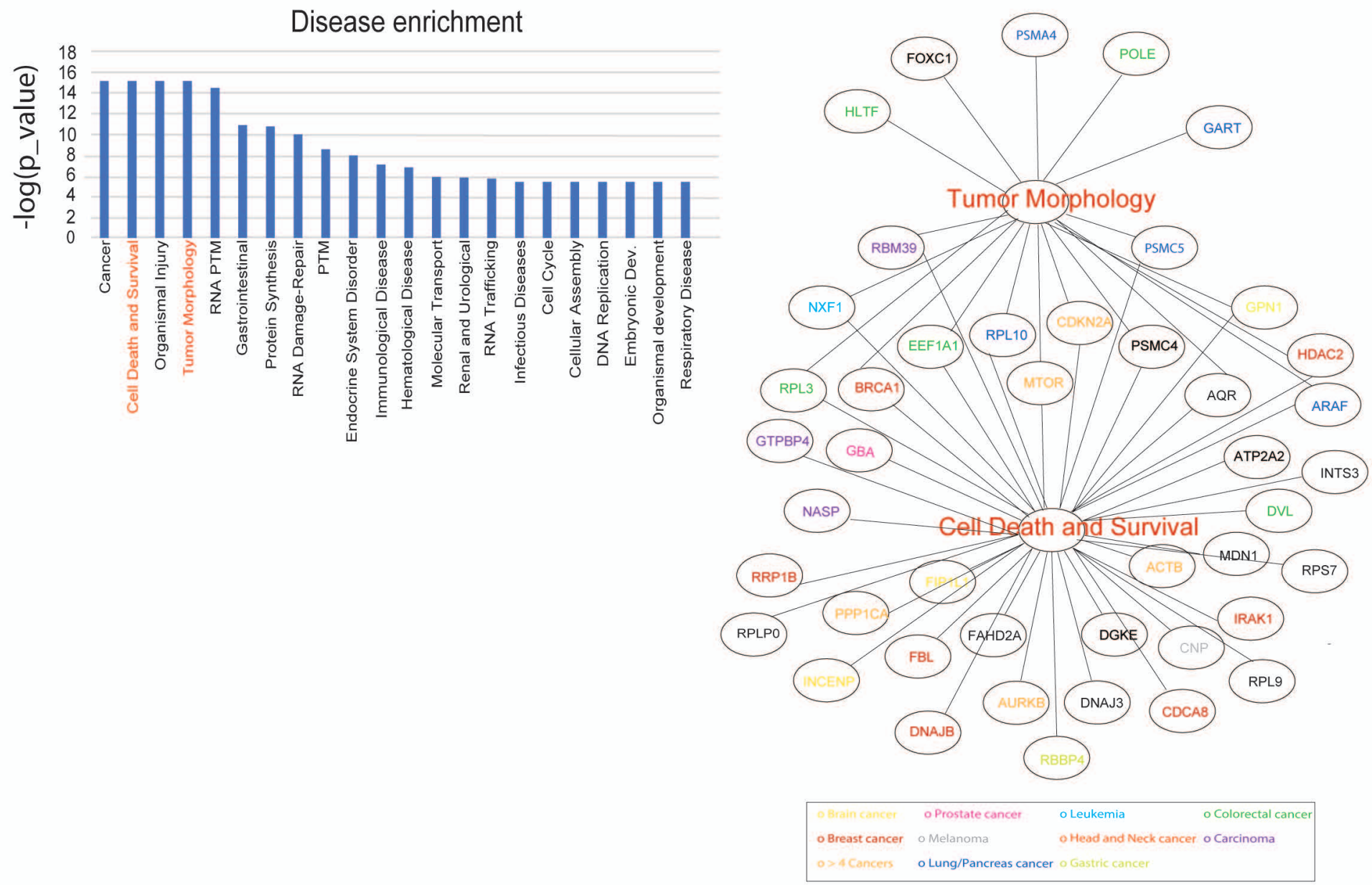

Figure 4. BRMS1 C-terminal binding partners are associated with known pathologies.

(A) Venn diagram illustrating the shared proteins between the four mutants. Proteins shared between the three mutants located on the C-terminal and the shared subunits between the S237D and BRMS1230-246 are indicated by a black box. (B) The Z-statistics and the TopS values of the 64 proteins shared between the mutants located on the C-terminal are illustrated in (B). T he red color corresponds to highest values (TopS or Z-statistics) whereas blue color indicates lower values. Proteins are colored in red if they are in the CRAPome database in less than $3 / 411$ controls with maximum 1 Spectral count. (C) Disease enrichment. Proteins showing significant changes in the mutants in the C-terminal were used in the IPA analysis to determine the most enriched diseases within these proteins. Top 10 enriched classes are displayed in the Figure 4C. (D) Proteins in the Tumor morphology class are illustrated in here. The proteins in the network were color coded according with the type of cancer they associate. 
$A$

BRMS1

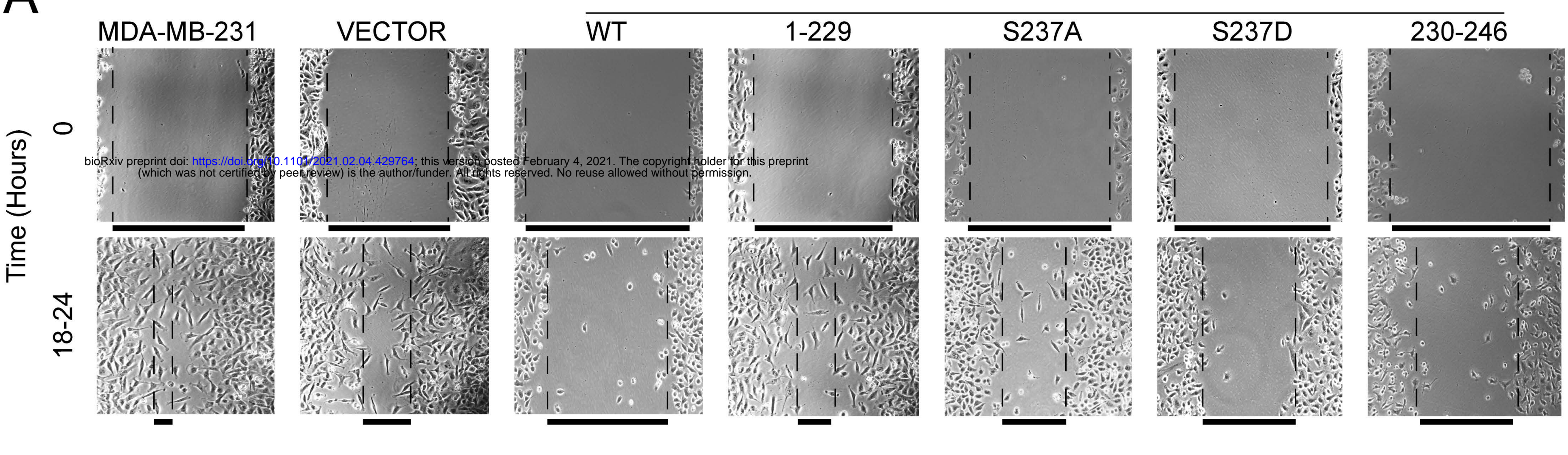

B

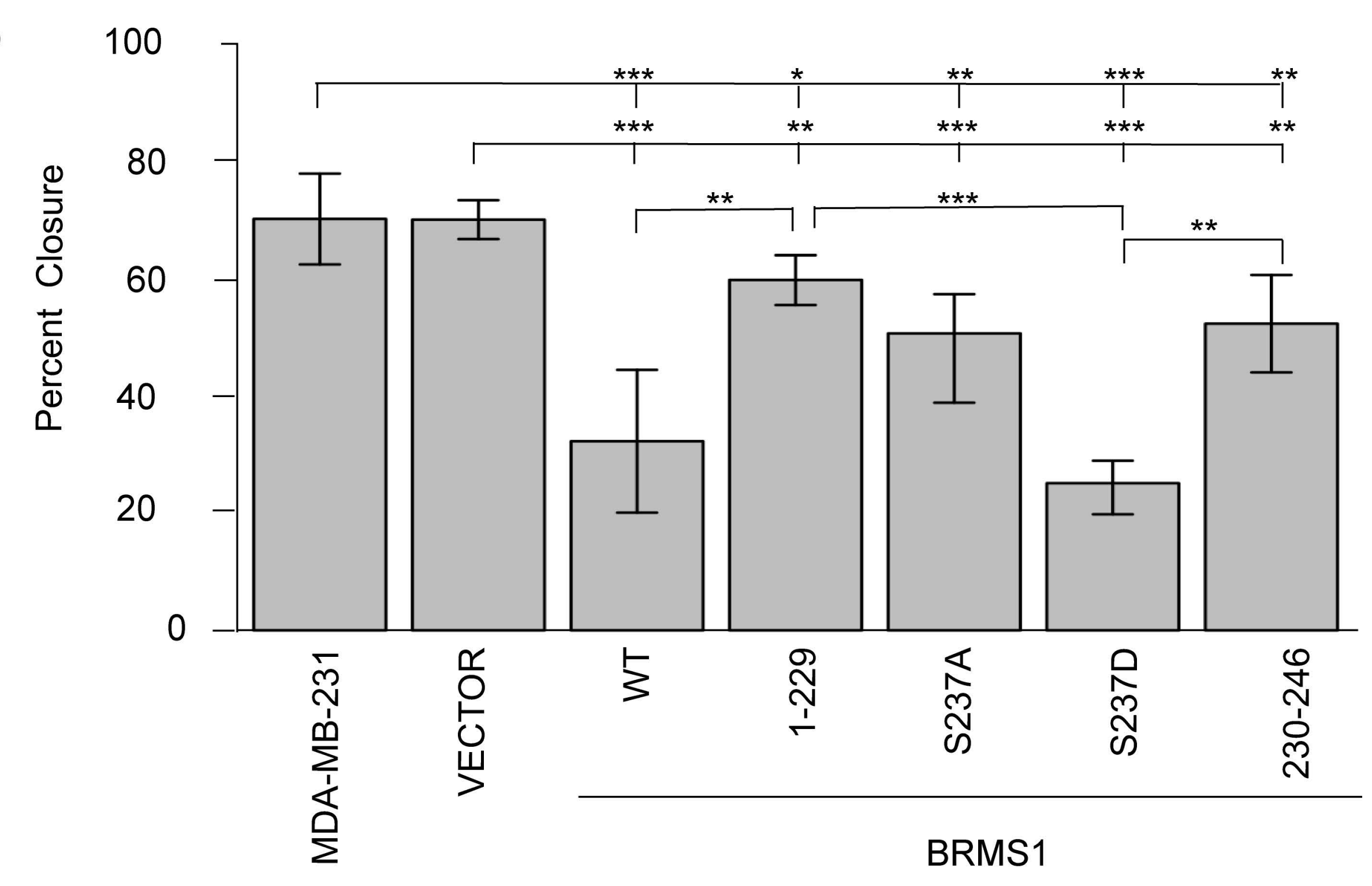

C

\begin{tabular}{|l|c|c|c|c|c|c|}
\hline & MDA-MB-231 & VECTOR & WT & $1-229$ & S237A & S237D \\
\hline VECTOR & 0.9 & & & & & \\
\hline WT & $0.00004^{* * *}$ & $0.000008^{* * *}$ & & & & \\
\hline $1-229$ & $0.04^{*}$ & $0.004^{* *}$ & $0.002^{* *}$ & & & \\
\hline S237A & $0.003^{* *}$ & $0.001^{* \star *}$ & 0.2 & 0.07 & & \\
\hline S237D & $0.000000007^{* * *}$ & $0.00000000004^{* * *}$ & 0.6 & $0.00001^{* * *}$ & 0.06 & \\
\hline 230-246 & $0.004^{* *}$ & $0.002^{* *}$ & 0.07 & 0.1 & 0.6 & $0.005^{* *}$ \\
\hline
\end{tabular}

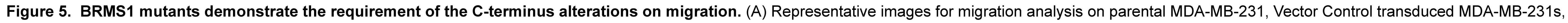

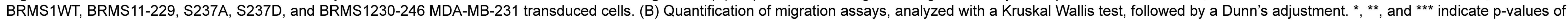
$\otimes 0.05, \otimes 0.01$, and $\otimes 0.001$, respectively. (C) This table includes the $p$-values for the comparisons completed in (B) 
Table 1. Predicted protein stability is impacted by $\mathbf{S 2 3 7}$ mutations.

\begin{tabular}{|c|c|c|} 
Mutant & Structure & $\begin{array}{c}\text { Stability } \\
\text { Changes }\end{array}$ \\
\hline S237A & Primary & Destabilizing \\
\hline S237A & Primary & Destabilizing \\
\hline
\end{tabular}

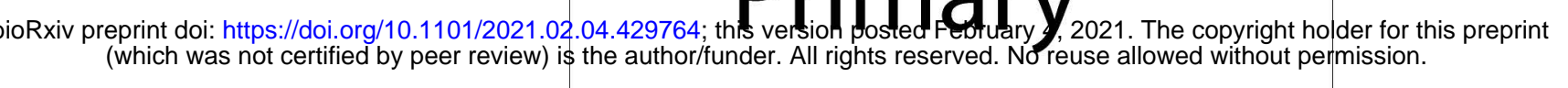

S237A

S237A

S237A

S237A

S237D

S237D

S237D

S237D

S237D

S237D
Tertiary

Primary

Tertiary

Primary

Primary

Primary

Tertiary

Primary

Tertiary

Primary
Destabilizing

Destabilizing

Destabilizing

Order $\rightarrow$

Disorder

Destabilizing

Destabilizing

Destabilizing

Destabilizing

Destabilizing

Order $\rightarrow$

Disorder
Software

Changes

MuProt

$-0.214633$

DDG:

$-1.69$

I-Mutant

Folding Rate:

$-1.1$

Folding Race

DDG:

$-0.095$

DynaMut

DD Encom:

$-0.292 \mathrm{kcal} / \mathrm{mol}$

DynaMut

DIM-Pred

DDG:

$-0.0552$

DDG:

$-1.0$

Folding Rate:

$-1.8$

DDG:

$-0.457$

MuProt

I-Mutant

Folding Race

DynaMut

DD Encom:

$-0.353$
DynaMut

DIM-Pred 

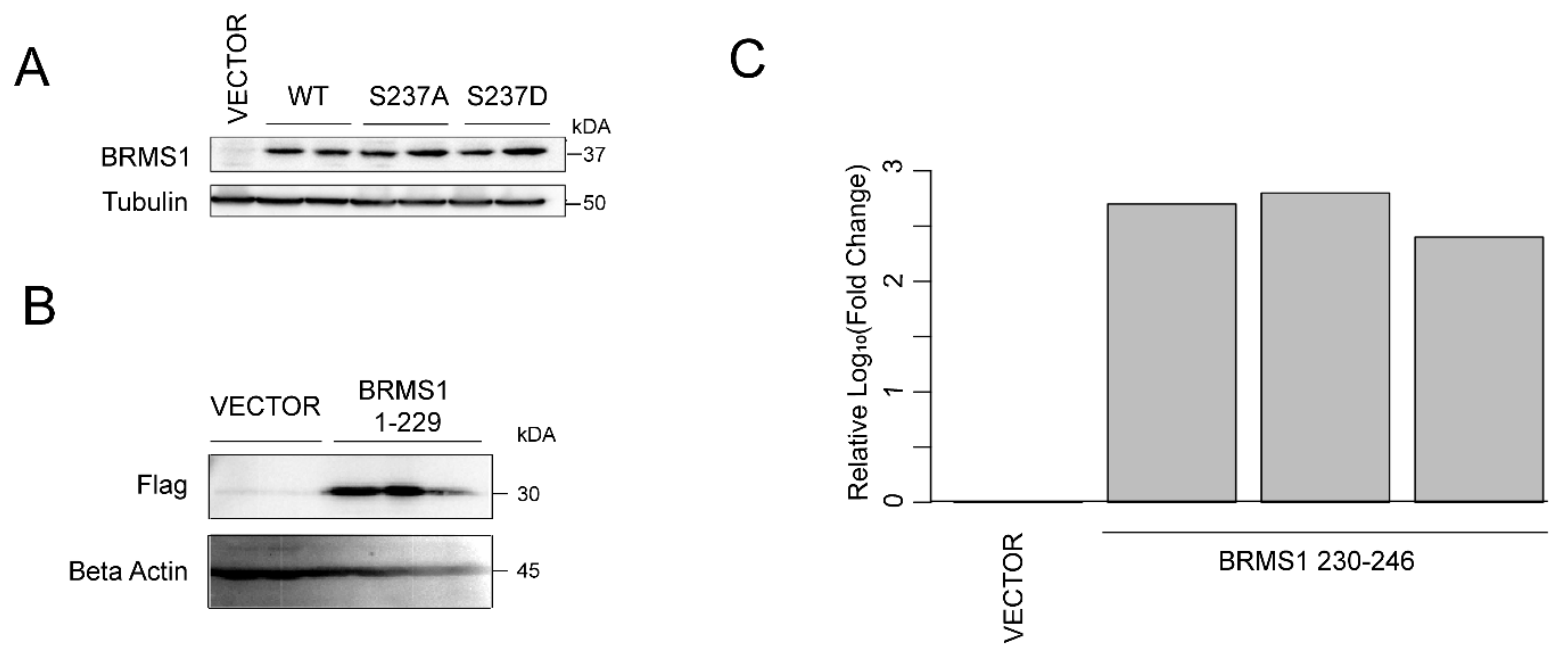

Supplemental Figure 1 Expression of BRMS1 mutants within MDA-MB-231 cells. (A) Expression of BRMS1 WT and phospho-mutants compared to Vector Control MDA-MB-231 cells. (B). Expression of BRMS1 $^{1-229}$ within MDA-MB-231 cells. (C) qPCR quantification of BRMS1 230-246 expression 


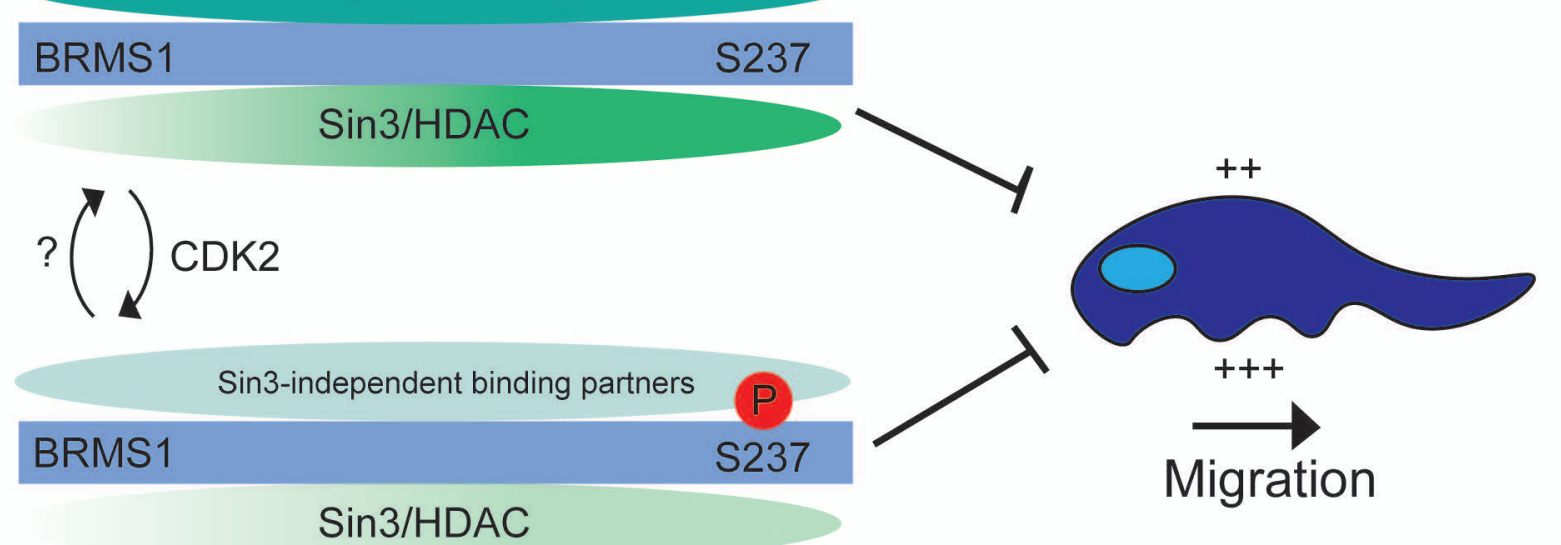

Graphical Abstract: Utilizing BRMS1 mutants to mimic-phosphorylation, this study demonstrates that S237-phosphorylation disrupts

BRMS1 protein-protein interactions. The disruption includes both known Sin3/HDAC interactors as well as additionally previously unidentified

Sin3-indepedent binding partners (indicated by increased opacity). It is revealed that BRMS1-phosphorylation status also more greatly inhibits cell migration (indicated by + ) compared to the unphosphorylated state, suggesting that phosphorylation plays a role in BRMS1 metastatsis suppresion function, potentially though altered protein interactions. 